\title{
SIGNIFICADO DO TRATAMENTO HOSPITALAR DE DESINTOXICAÇÃO PARA PESSOAS COM ALCOOLISMO: RETOMANDO A VIDA*
}

\author{
Jucimara da Silva Mendes ${ }^{1}$, Lucas Corrêa Preis², Débora FelippeBrolese', \\ José Luís Guedes dos Santos ${ }^{3}$, Greice Lessa ${ }^{4}$
}

\begin{abstract}
RESUMO: Objetivo: compreender o significado do tratamento hospitalar de desintoxicação para pessoas com alcoolismo. Método: pesquisa qualitativa, com a Teoria Fundamentada nos Dados como referencial metodológico. A amostragem teórica foi composta por 22 participantes, incluindo usuários de álcool internados em uma clínica psiquiátrica do Sul de Santa Catarina, profissionais de saúde da instituição e familiares de usuários em tratamento. Os dados foram coletados no segundo semestre de 2015, por meio de entrevistas, e analisados mediante codificação inicial e focalizada. Resultados: o fenômeno obtido intitula-se "Retomando a vida após tratamento hospitalar de desintoxicação", que é sustentado pelas categorias: Iniciando o vício pelo álcool; Perdendo o controle sobre o vício do álcool; Buscando o tratamento hospitalar de desintoxicação; Percebendo os benefícios do tratamento; e, preparando-se para a alta hospitalar. Conclusão: o tratamento hospitalar de desintoxicação representa uma oportunidade das pessoas com alcoolismo retomarem as suas vidas e reinserirem-se na sociedade de modo produtivo.
\end{abstract}

DESCRITORES: Alcoolismo; Saúde Mental; Cuidados de Enfermagem; Pesquisa Qualitativa; Reabilitação.

\section{MEANING OF DETOXIFICATION IN A HOSPITAL SETTING FOR ALCOHOL-DEPENDENT PEOPLE: RESUMING LIFE}

ABSTRACT: Objective: To understand the meaning of detoxification for alcohol-dependent people in a hospital setting. Method: Qualitative study based on the Grounded Theory methodology. The theoretical sample consisted of 22 participants, including alcohol users admitted to a psychiatric clinic in the southern region of the state of Santa Catarina, health professionals from the institution and family members of users under treatment at the clinic. Data was collected in the second half of 2015 through interviews and analyzed using initial and focused codes. Results: The identified phenomenon is called "Resuming life after alcohol detoxification in a hospital setting". Conclusion: Detoxification in a hospital setting provides an opportunity for alcohol-dependent people to resume their lives and reintegrate themselves into society in a productive way.

KEYWORDS: Alcoholism; Mental Health; Nursing Care; Qualitative Study; Rehabilitation.

\section{SIGNIFICADO DEL TRATAMIENTO HOSPITALARIO DE DESINTOXICACIÓN PARA PERSONAS CON ALCOHOLISMO: RETOMANDO LA VIDA}

RESUMEN: Objetivo: comprender el significado del tratamiento hospitalario de desintoxicación para personas con alcoholismo. Método: investigación cualitativa, por medio de la Teoría Fundamentada en los Datos como referencial metodológico. Veintidós personas participaron de la muestra teórica, incluyéndose usuarios de alcohol internados en una clínica psiquiátrica del Sur de Santa Catarina, profesionales de salud de la institución y familiares de usuarios en tratamiento. Se recogieron los datos en segundo semestre de 2015, por medio de entrevistas, y se los analizaron por codificación inicial y focalizada. Resultados: el fenómeno obtenido se titula "Retomando la vida después del tratamiento hospitalario de desintoxicación", y está basado en las categorías: Iniciando la adicción por el alcohol; Perdiendo el control sobre la adicción al alcohol; Buscando el tratamiento hospitalario de desintoxicación; Percibiendo los beneficios del tratamiento; y, Preparándose para el alta hospitalario. Conclusión: el tratamiento hospitalario de desintoxicación representa una oportunidad para las personas con alcoholismo de retomar sus vidas y de volver a la sociedad de modo productivo.

DESCRIPTORES: Alcoholismo; Salud Mental; Cuidados de Enfermería; Investigación cualitativa; Rehabilitación.

\footnotetext{
*Artigo extraído do Trabalho de Conclusão de Curso de Graduação em Enfermagem intitulado: O alcoolismo e a desintoxicação hospitalar: a visão do usuário. Centro Universitário Barriga Verde, 2015.

'Enfermeira. Pós-graduanda em Urgência e Emergência. Centro Universitário Barriga Verde. Orleans, SC, Brasil.

${ }^{2}$ Enfermeiro. Mestrando em Enfermagem pela Universidade Federal de Santa Catarina. Florianópolis-SC-Brasil.

${ }^{3}$ Enfermeiro. Doutor em Enfermagem. Docente do Departamento de Enfermagem. Universidade Federal de Santa Catarina. Florianópolis, SC, Brasil.

${ }^{4}$ Enfermeira. Doutoranda em Enfermagem. Universidade Federal de Santa Catarina. Florianópolis, SC, Brasil.
} 


\section{INTRODUÇÃO}

O consumo de álcool é uma característica cultural da sociedade e está associado à busca de prazer, à compensação de medos, à comemoração de conquistas e ao alívio do estresse e/ou de dores físicas. Porém, quando o uso dessa substância torna-se repetitivo e leva o indivíduo à perda do autocontrole, gerando impactos na sua vida social, familiar, profissional, bem como na saúde física e mental, caracteriza-se uma dependência química. ${ }^{(1-2)}$

Nessa perspectiva, considera-se que os danos causados aos jovens, em relação ao uso abusivo de álcool, são diferentes em relação aos adultos. O perfil do jovem alcoolista é voltado para grandes desordens, ocasionando principalmente riscos para si, prejudicando a fase de desenvolvimento em que se encontra e, posteriormente, afetando os demais. O consumo abusivo do álcool e outras substâncias estão relacionados com os diversos fatores que influenciam, direta e indiretamente, na qualidade de vida desses jovens, com destaque para fatores individuais, micro e macrossociais. Portanto, ações devem ser realizadas com atenção ao desenvolvimento pessoal e nível de informação adequados, evitando futuros danos físicos, mentais e sociais. ${ }^{(3-4)}$

Globalmente, considera-se que a cada ano cerca de dois bilhões de pessoas consomem bebidas alcoólicas, o que corresponde a aproximadamente $40 \%$ da população mundial acima de 15 anos. Assim, o consumo do álcool é um dos fatores de risco de maior impacto para a morbidade, mortalidade e incapacidades em todo o mundo. Estimativas indicam que morrem em torno de 3,3 milhões de pessoas a cada ano devido ao uso nocivo de álcool no mundo, ou seja, quase $6 \%$ das mortes são atribuídas total ou parcialmente ao álcool. As faixas etárias jovens (20-49 anos) são as principais atingidas pelas mortes associadas ao consumo do álcool, representando perda expressiva de pessoas economicamente ativas. ${ }^{(5)}$

No Brasil, o consumo de álcool estimado é equivalente a 8,7 litros por pessoa a cada ano, que é superior à média mundial de 6,2 litros. É um dos países que lidera o ranking do consumo abusivo de álcool de uma só vez, sendo considerado o consumo de 60 gramas ou mais de álcool puro, pelo menos uma vez por mês. Estima-se que consumo abusivo de álcool atinge 16,5\% da população brasileira com idade igual ou superior aos 18 anos. As principais doenças ou prejuízos associados ao álcool são violência interpessoal, cirrose hepática, câncer de esôfago, síndrome alcoólica fetal e pancreatite. Dessa forma, as consequências do uso de álcool oneram a sociedade, direta e indiretamente, aumentando os custos dos serviços de saúde e do sistema previdenciário. Além disso, o álcool também está associado ao aumento do absenteísmo e à diminuição da produtividade no trabalho. ${ }^{(5)}$

Diante da magnitude do problema de consumo abusivo de bebidas alcoólicas, o Ministério da Saúde brasileiro, desde 2003, por meio do programa Atenção Integral a Usuários de Álcool e outras Drogas, tem desenvolvido ações e políticas no intuito de prevenir, tratar e reabilitar os usuários de álcool e outras drogas. Além disso, várias campanhas publicitárias têm sido vinculadas visando à prevenção e diminuição do uso bebidas alcoólicas. ${ }^{(1)}$ Portanto, nos serviços de saúde, a dependência do álcool precisa ser abordada não somente pela ótica psiquiátrica ou médica, mas, sobretudo, pelas suas implicações sociais, psicológicas, econômicas e políticas, considerando os múltiplos fatores relacionados com uma dependência química. ${ }^{(1,6)}$

Para iniciar um tratamento contra a dependência, uma das opções dos usuários de álcool é a internação hospitalar. O tratamento hospitalar de desintoxicação configura-se como internação de curta duração até a estabilidade clínica do usuário. Após a alta hospitalar, busca-se a inserção do individuo nos serviços da Rede de Atenção Psicossocial. ${ }^{(5)}$ Trata-se de um momento delicado na vida do dependente químico, pois o usuário permanece fora de seu habitat natural, longe de família e amigos por um período. Nesse momento, os vínculos com a equipe de saúde podem colaborar para o sucesso do tratamento, tanto durante o processo de desintoxicação como após a alta hospitalar. ${ }^{(6)}$

Dessa forma, considerando o cenário epidemiológico que revela o elevado consumo abusivo de álcool no Brasil e a escassa produção científica de Enfermagem sobre o tema alcoolismo, ${ }^{(1,7)}$ principalmente diante da relevância clínica e social dessa problemática, o presente estudo teve como questão de pesquisa: Qual é o significado do tratamento hospitalar de desintoxicação para pessoas com alcoolismo?

O objetivo do estudo foi compreender o significado do tratamento hospitalar de desintoxicação para pessoas com alcoolismo. 


\section{- MÉTODO}

Trata-se de um estudo qualitativo, cujo referencial metodológico foi a perspectiva construtivista da Teoria Fundamentada nos Dados (TFD). ${ }^{(8)}$

O local de estudo foi uma clínica psiquiátrica hospitalar com 25 leitos, localizada em um município do Sul de Santa Catarina, com aproximadamente 20 mil habitantes. O corpo clínico da instituição é formado por uma enfermeira, cinco técnicos de enfermagem, um médico clínico geral, um médico psiquiatra, uma nutricionista, um assistente social, um psicólogo e uma farmacêutica.

A amostragem teórica foi obtida por meio de três grupos amostrais (GA), perfazendo um total 22 participantes, conforme apresentado no Quadro1.

Quadro 1- Amostragem teórica. Orleans, SC, Brasil, 2015

\begin{tabular}{cll}
\hline & Grupos amostrais & Objetivos \\
\hline GA1 & $\begin{array}{l}\text { 10 usuários de álcool internados na clínica de } \\
\text { reabilitação (P1-P10) }\end{array}$ & $\begin{array}{l}\text { Compreender o desenvolvimento do vicio pelo álcool e o } \\
\text { significado do tratamento hospitalar de desintoxicação. }\end{array}$ \\
\hline GA2 & $\begin{array}{l}\text { 05 usuários de álcool internados na clínica } \\
\text { entre 20 e 30 dias (P11-P15) }\end{array}$ & $\begin{array}{l}\text { Analisar se a visão dos usuários no final do tratamento era a } \\
\text { mesma referida no início da internação. }\end{array}$ \\
\hline GA3 participantes, sendo 04 profissionais de saúde & $\begin{array}{l}\text { (02 técnicos de enfermagem, 01 psicólogo, 01 } \\
\text { assistente social) e 03 familiares de pacientes a importância do suporte profissional e familiar } \\
\text { internados na instituição (P16-P22) }\end{array}$ & $\begin{array}{l}\text { para o tratamento, bem como a relação dos profissionais e } \\
\text { familiares com os usuários. }\end{array}$ \\
\hline
\end{tabular}

A composição dos grupos amostrais e o tamanho da amostragem teórica foram determinados pela saturação teórica dos dados. ${ }^{(8)}$ A coleta dos dados ocorreu entre agosto e novembro de 2015, por meio de entrevista intensiva, composta por questões abertas. ${ }^{(8)}$

As entrevistas foram gravadas, tiveram duração média de 25 minutos e foram transcritas na íntegra. Para as pessoas com alcoolismo, as questões versaram sobre oinício do consumo de álcool, a existência de história familiar de consumo de álcool, os significados e as expectativas com o tratamento, as dificuldades durante a desintoxicação e o papel da família no tratamento. As questões do segundo grupo amostral seguiram o mesmo roteiro do primeiro grupo, porém, com foco na fase final do tratamento. Os profissionais e familiares foram questionados sobre a sua participação no tratamento hospitalar de desintoxicação das pessoas com alcoolismo.

A análise dos dados foi realizada com auxílio do software NVIVOß em duas etapas: codificação inicial e focalizada. ${ }^{(8)}$ A codificação inicial consistiu na análise dos fragmentos dos dados, identificando incidentes e transformando-os em códigos. Na codificação focalizada, os códigos anteriores mais significativos e/ou mais frequentes foram agrupados até gerar um fenômeno ou categoria central, sustentado por categorias e subcategorias. ${ }^{(8)}$ As categorias e o fenômeno obtidos foram validados por um grupo de pesquisadores com experiência na área de saúde mental e/ou no método da TFD.

Quanto aos aspectos éticos, os participantes assinaram o Termo de Consentimento Livre e Esclarecido (TCLE) e a pesquisa foi aprovada pelo Comitê de Ética em Pesquisa de referência, sob parecer n..$^{\circ}$ 1.165.995. Os depoimentos foram identificados com códigos compostos pela letra " $\mathrm{P}$ " e números conforme a ordem das entrevistas: P1, P2,..., P22. 


\section{RESUlTADOS}

As cinco categorias que emergiram da análise dos dados estão apresentadas no Quadro 2. A partir da interconexão das categorias, obteve-se o fenômeno "Retomando a vida após tratamento hospitalar de desintoxicação". A seguir, descreve-se cada uma das categorias.

Quadro 2- Categorias e fenômeno. Orleans, SC, Brasil, 2015

\begin{tabular}{|c|c|}
\hline Categorias & Fenômeno \\
\hline 1. Iniciando o vício pelo álcool & Retomando a vida após o tratamento \\
\hline 2. Perdendo o controle sobre o vício do álcool & hospitalar de desintoxicação \\
\hline 3. Buscando o tratamento hospitalar de desintoxicação & \\
\hline 4. Percebendo os benefícios do tratamento & \\
\hline 5. Preparando-se para a alta hospitalar & \\
\hline
\end{tabular}

\section{Iniciando o vício pelo álcool}

O consumo de álcool entre os usuários teve início, principalmente, na adolescência, durante momentos de lazer e a partir da influência de amigos. A família também influencia para o início do uso do álcool, pois a mãe e pai de alguns usuários eram alcoolistas. O uso abusivo do álcool também foi favorecido pela vivência de momentos traumáticos, como: perda de um familiar, desemprego e término de relacionamento amoroso.

Eu comecei jovem apenas por diversão, por causa da idade adolescente, em festas com amigos [...]. (P1)

Meu pai era alcoólatra, já fui influenciado por ele. [...] eu era pequeno e ele já me acordava para fazer bagunça e beber junto. (P2)

Tudo começou com o falecimento do meu filho, foi quando comecei a beber todo dia. (P8)

\section{Perdendo o controle sobre o vício}

O aumento da frequência e quantidade de álcool consumido levaram os indivíduos à perda do controle sobre dessa substância, tornando-os dependentes. As pessoas não percebiam inicialmente a dependência, inventavam desculpas para si e aumentavam cada vez mais o consumo de álcool por variados motivos, entre os quais foram citados o lazer, a timidez e solidão.

Eu saia de manhã e sempre procurava um pretexto e ia para o bar, chegava em casa, às vezes, 2 horas da tarde [...] só arrumava um pretexto, só para sair e passar nos bares para tomar.(P9)

Eu bebia para dar em cima das gurias, senão, não chego perto de mulher. (P6)

Assim, o vício instalou-se de maneira progressiva. Alguns usuários relataram manter controle do vício por meio de tratamento médico, permanecendo períodos sem beber. Porém, as recaídas eram frequentes, o que os levava novamente ao consumo exagerado.

Eu fiz tratamento, fiquei três anos sem beber. Depois recai e voltei a beber. Depois paguei consulta particular, fiquei mais seis meses sem beber, mas recai e voltei de novo. (P9)

No início eu bebia, mas nada que atrapalhasse minha vida, meu estudo, não era uma coisa compulsiva. Mas, ao longo do tempo, foi indo e perdi o controle. (P1)

Ao longo do tempo, o vício pelo álcool comprometeu o desempenho profissional dos usuários e gerou conflitos familiares. Tais repercussões negativas fizeram com que as pessoas compreendessem o vício pelo álcool como uma doença, a qual necessita de tratamento. 
Já trabalhei em vários lugares, como em oficinas mecânicas. Porém, sempre mudando de emprego por conta da bebida. (P2)

O álcool me prejudicou muito. Foi responsável por brigas e conflitos com familiares, porque querendo ou não o álcool altera o humor. (P7)

O alcoolismo é uma doença, [...] a pessoa começa a beber e depois, muitas vezes, não consegue mais se controlar e acaba, muitas vezes, se afundando no alcoolismo. (P18)

\section{Buscando o tratamento hospitalar de desintoxicação}

Essa categoria marca o momento no qual o dependente de álcool percebe que precisa de ajuda e inicia um tratamento hospitalar para desintoxicação alcoólica. O dependente químico busca a internação não somente para obter ajuda profissional, mas também como uma maneira de se afastar da sociedade e, consequentemente, do álcool. Nessa etapa, destacou-se a importância do apoio e incentivo de amigos e familiares para o começo do tratamento.

Eu precisava de um tempo sozinho, sem influência do meu ambiente, do meu habitat natural. Distância para poder refletir. (P10)

Tive apoio dos amigos [...] teve muita gente que me apoiou. O próprio dono do bar que eu ia me deu força e veio me visitar aqui. (P15)

Amo a minha família toda, eles que correram atrás de tudo para mim [...] toda família está me apoiando e eu estou muito faceiro com isso. (P7)

A fase inicial do processo de tratamento hospitalar desintoxicação é fundamental para o sucesso do tratamento. Porém, também é o momento mais difícil para os usuários, devido a alterações orgânicas, físicas e psicológicas oriundas de crises de abstinência do álcool. Nesse momento, algumas pessoas chegam a questionar se precisam mesmo de tratamento.

No começo, é complicado! A gente passa por uma fase de desintoxicação, mais é necessária, e ai muitos pacientes tem crises de ansiedade. (P14)

Eu não sei se preciso de ajuda, sei lá, se eu caprichasse mesmo, o importante é ter força de vontade própria. (P2)

\section{Percebendo os benefícios do tratamento}

Ao longo do tratamento hospitalar de desintoxicação, os usuários passaram a se sentir confiantes e engajados na sua recuperação. O bom convívio com os colegas de internação foi destacado como um fator positivo para isso, assim como as normas e rotinas estabelecidas pela instituição.

Estou bem melhor e confiante que vou sair daqui forte para encarar o mundo lá fora [...] cada dia é uma experiência nova, vou ficar até o final para garantir que não tenha recaída. (P14)

[...] temos várias regras e horários que para muitos, na vida ativa bebendo lá fora, não tem mais essas regras e horários, não cumpre mais um horário certo para almoço, para iniciar no trabalho e terminar, [...] tudo isso é focado aqui no tratamento. (P7)

Já vi que melhorei, eu estava muito ansioso e nervoso e isso já melhorou um pouco [...]. Eu peguei várias funções aqui dentro, a gente vai fazendo um serviço daqui e outro ali, e vamos adaptando e esquecendo $[\ldots .$.$] , isso fez a minha autoestima subir. (P13)$

\section{Preparando-se para a alta hospitalar}

Após passar pelo processo hospitalar de desintoxicação, as pessoas com alcoolismo precisam retomar sua vida e lidar com as situações diárias que podem favorecer recaídas. Vários são os sentimentos e 
interrogações que surgem na vida do usuário no momento da alta hospitalar. Muitos saem confiantes, acreditando no sucesso do tratamento. Porém, o medo e ansiedade também estão presentes. Enquanto estão internados, eles não têm acesso ao álcool e de volta à sociedade precisarão de estratégias para lidar com o vício e evitar recaídas.

Eu quero melhorar, saindo daqui eu vou tentar evitar o máximo possível ir a bares e essas coisas, vou aguardar um mês certinho e voltar para a casa. (P2)

É um tratamento longo e eu vou ter que me adaptar no dia-a-dia lá fora, porque aqui dentro é uma coisa e lá fora é outra. [...] espero mudar e não decepcionar mais as minhas filhas. (P13)

Eu tenho medo é lá de fora, aqui dentro eu não tenho medo. Lá fora é que vêm as dificuldades. (P4)

Os usuários deixam a instituição hospitalar, conscientes de que a fase de manutenção do tratamento é tão importante quanto o processo de desintoxicação. Nesse sentido, destacaram que irão frequentar os grupos de Alcoólicos Anônimos (AA) e os Centros de Atenção Psicossocial (CAPS). Um dos profissionais entrevistados pontuou que uma das dificuldades para a continuidade do tratamento é a falta de uma rede de serviços de saúde resolutivos. O usuário de álcool sai da internação hospitalar e, muitas vezes, perde o vínculo com os serviços de saúde, o que dificulta a continuidade do tratamento.

A internação muda muita coisa, quando eu sair daqui, a primeira coisa que vou fazer é frequentar o $A A$ e o CAPS. E quero trabalhar no meu ramo [...]. (P12)

Há uma dificuldade de ter um tratamento ambulatorial adequado após sua alta, uma vez que as redes de atendimento psicossocial ainda não estão presentes em todos os municípios, e quando estão os profissionais não se sentem preparados para atender esse tipo de paciente. (P19)

\section{- DISCUSSÃO}

Os achados deste estudo confirmam resultados de pesquisas anteriores, ${ }^{(3,5-6,9)}$ segundo as quais o início do uso do álcool acontece principalmente na adolescência por influência de amigos e familiares. Um estudo realizado no Rio de Janeiro, Brasil, revelou que os adolescentes estão experimentando de forma cada vez mais precoce, bebidas alcoólicas, devido ao fácil acesso e à curiosidade típica dessa faixa etária que os fazem buscar por novas experiências e sensações. ${ }^{(7)}$ Além disso, o convívio com dependentes químicos entre os integrantes da família faz com que crianças e adolescentes tenham contato com bebidas alcoólicas precocemente, o que pode motivá-las seguir as referências e condutas observadas no contexto familiar. ${ }^{(9-10)}$

Em consonância com os resultados da presente pesquisa, a literatura apresenta que o consumo em excesso do álcool também tem sido relacionado com fatores e situações traumáticas, tais como: depressão, estresse, ansiedade, obesidade, problemas conjugais e perda de um ente querido. ${ }^{(11-12)}$ Estudo desenvolvido na Índia com mulheres usuárias de álcool e outras drogas evidenciou que o início do vício esteve associado à perda do marido, busca por prazer e supressão da dor emocional. ${ }^{(13)}$

Além da facilidade de acesso ao álcool e a ocorrência de situações traumáticas, estressantes e/ou de solução difícil, é importante salientar que o hábito de consumir bebidas alcoólicas é visto como um comportamento social vinculado à própria cultura da população no Brasil, o qual é reforçada pela mídia. ${ }^{(3-4)}$ Esse conjunto de fatores pode levar os indivíduos à perda do controle sobre o vício, conforme apresentado na segunda categoria do estudo.

Os usuários de álcool, na maioria das vezes, apresentam várias tentativas de tratamento e permanecem alguns períodos em abstinência. Porém, a vontade de usar álcool torna-se cada vez mais persistente, o que acaba levando novamente ao consumo. ${ }^{(14)}$ A busca pela internação hospitalar para desintoxicação alcoólica acontece quando os usuários percebem-se imersos em um contexto de vida marcado por dificuldades profissionais e conflitos familiares, bem como agravos biológicos e psicológicos nos usuários. ${ }^{(15-16)}$ Esse pode ser considerado o momento em que o dependente de álcool aceita a sua doença e reconhece que precisa de ajuda para livrar-se do vício. ${ }^{(17)}$ 
O dependente químico busca a internação, muitas vezes, não somente para obter ajuda profissional, mas também como uma maneira de manter-se afastado da sociedade e consequentemente do álcool. A fase inicial do processo hospitalar de desintoxicação mostrou-se como a base da manutenção do tratamento. Porém, esse aspecto deve ser trabalhado junto ao dependente químico, pois no momento em que ele volta a se inserir na sociedade o álcool estará presente também em sua vida, tornando-se necessário desenvolver estratégias para manter-se firme no tratamento e evitar recaídas.

Assim, pontua-se que os serviços de saúde mental devam oferecer tratamento terapêutico convergente às necessidades dos dependentes, fortalecendo a adesão integral ao tratamento e evitando a evasão ou abandono da unidade de tratamento sem a finalização do tratamento. ${ }^{(18)} \mathrm{O}$ apoio dos familiares e dos amigos foi considerado primordial para o alcance do sucesso no tratamento da dependência e para a manutenção da abstinência, corroborando resultados de investigação anterior. ${ }^{(18)} \mathrm{O}$ apoio familiar foi considerado um fator motivacional para o sucesso do tratamento de desintoxicação entre usuários de álcool e outras drogas de Berlim, Alemanha. ${ }^{(19)} \mathrm{O}$ suporte dos amigos também é uma fonte de motivação para o enfrentamento das mudanças e as dificuldades iniciais do tratamento. ${ }^{(9,11)}$

Outro aspecto valorizado pelos usuários no tratamento hospitalar foi o desenvolvimento de relações amistosas com os colegas de internação. No tratamento da dependência química, amizades entre os usuários possibilitam o desenvolvimento de estratégias coletivas de enfrentamento das dificuldades vivenciadas durante a internação hospitalar, como a solidão e as crises de abstinência. ${ }^{(2,20)}$ De modo semelhante, estudo realizado na Inglaterra também ressaltou a importância do apoio e da ajuda mutua entre usuários em processo de desintoxicação alcoólica. ${ }^{(21)}$

Em relação ao contexto da internação hospitalar, destacou-se a organização de uma rotina para os usuários, com definição de horários e atividades a serem desenvolvidas ao longo do dia. O cumprimento de regras e rotinas é importante para o tratamento da dependência química, pois promove o comprometimento e a responsabilização dos pacientes com atividades da vida diária. Também se configura como um exercício de adaptação para a alta hospitalar e reinserção social dos pacientes. ${ }^{(17)}$

Quando o usuário chega à fase final do tratamento, percebe que consegue manter sob controle o vício pelo álcool e sente-se confiante para reintegrar sua a vida social e familiar. ${ }^{(2)}$ Porém, pesquisadores norte-americanos destacam que a manutenção de vínculos terapêuticos com os serviços de saúde é fundamental para a continuidade do tratamento do uso abusivo do álcool após a alta hospitalar. ${ }^{(22)}$ Entre os programas e os serviços de saúde mental no Brasil, o Centro De Atenção Psicossocial Álcool e Drogas (CAPSad) tem um papel importante na reabilitação do alcoolista, após o processo hospitalar de desintoxicação. ${ }^{(23-24)}$

Nesse sentido, é importante mencionar a preocupação expressada por um dos profissionais, acerca da organização e estruturação da rede de serviços de saúde mental para a continuidade do tratamento no contexto investigado. Portanto, considera-se relevante que sejam discutidas estratégias pelos profissionais e gestores, visando à inserção e ao acompanhamento dos usuários após a alta hospitalar em serviços da rede de saúde mental.

\section{- CONCLUSÃO}

A partir do fenômeno "Retomando a vida após tratamento hospitalar de desintoxicação", este estudo possibilitou a compreensão de que o tratamento hospitalar de desintoxicação representa uma oportunidade das pessoas com alcoolismo retomarem as suas vidas e reinserirem-se na sociedade de modo produtivo.

O desenvolvimento do vicio pelo álcool e o significado do tratamento hospitalar de desintoxicação para pessoas com alcoolismo foram apresentados por meio de cinco categorias: Iniciando o vício pelo álcool; Perdendo o controle sobre o vício do álcool; Buscando o tratamento hospitalar de desintoxicação; Vendo os benefícios do tratamento de desintoxicação hospitalar; e, Preparando-se para a alta hospitalar.

Esses resultados fornecem subsídios para a prática de profissionais de enfermagem e saúde que atuam no cuidado de pessoas em tratamento do vício do alcoolismo. O entendimento do processo de 
desintoxicação, vivenciado pelas pessoas com alcoolismo, é importante para o desenvolvimento de estratégias que possam auxiliá-las no controle do vicio tanto durante o tratamento quanto na fase de manutenção.

A limitação da pesquisa refere-se à abrangência da coleta de dados, pois os dados foram obtidos a partir da realidade de uma clínica psiquiátrica hospitalar. Assim, sugere-se que novos estudos sejam realizados incluindo outros cenários de cuidado. Além disso, pontua-se a necessidade de uma nova investigação sobre o processo de reinserção social das pessoas, após o tratamento de desintoxicação alcoólica.

\section{- REFERÊNCIAS}

1.Silva SED, Padilha MI, de Cunha NMF, Vasconcelos EV. O alcoolismo nas pesquisas da enfermagem brasileira. Revista Gestão \&Saúde.[Internet]2015;6(3) [acesso em 24jan 2017]. Disponível: http://dx.doi.org/10.18673/gs.v6i3.22418.

2. Ponce TD, Prates JG, de Vargas D, de Oliveira MAF, Claro HG, Gnatta LR. Treinamento de equipes de enfermagem para assistência à síndrome de abstinência alcoólica: revisão integrativa. SMAD, Rev. eletrônica saúde mental alcooldrog. [Internet]2016;12(1) [acesso em 25jan 2017]. Disponível:http://dx.doi.org/10.11606/issn.1806-6976.v12i1p58-64.

3. Brito I, Precioso JAG, Correia C, Albuquerque C, Samorinha C, Cunha-Filho H, et al. Fatores associados ao consumo de álcool na adolescência, em função do gênero. Psic., saúde \&doenças. [Internet] 2015;16(3) [acesso em 23 jan 2017]. Disponível: http://dx.doi.org/10.15309/15psd1603010.

4. Castaño-Perez GA, Calderon-Vallejo GA. Problemas associados ao consumo de álcool em estudantes universitários. Rev. Latino-Am. Enfermagem. [Internet] 2014;22(5) [acesso em 24 jan 2017]. Disponível: http://dx.doi.org/10.1590/01041169.3579.2475.

5. World Health Organization. 66th World Health Assembly: Global status report on alcohol and health. Geneva: World Health Organization; 2014. 376p. Relatório final.

6.da Silva ML, Guimarães CF, Salles DB. Fatores de risco e proteção à recaída na percepção de usuários de substâncias psicoativas. Rev. Rene. [Internet]2014;15(6) [acesso em 27 set 2015].Disponível: http://dx.doi.org/10.15253/21756783.2014000600014.

7. Ministério da Saúde (BR). Portaria no 148, de 31 de janeiro de 2012. Define as normas de funcionamento e habilitação do Serviço Hospitalar de Referência para atenção a pessoas com sofrimento ou transtorno mental e com necessidades de saúde decorrentes do uso de álcool, crack e outras drogas, do Componente Hospitalar da Rede de Atenção Psicossocial e institui incentivos financeiros de investimento e de custeio. Diário Oficial da União [Internet] 31 jan 2012 [acesso em 22fev2017]. Disponível: http://bvsms.saude.gov.br/bvs/saudelegis/gm/2012/prt0148_31_01_2012.html.

8. CharmazKA. A construção da teoria fundamentada: guia prático para análise qualitativa. Porto Alegre: Artmed; 2009.

9. Neves KC, Teixeira MLO, Ferreira MA. Fatores e motivação para o consumo de bebidas alcoólicas na adolescência. Esc. Anna Nery. [Internet] 2015;19(2) [acesso em 21jan 2017]. Disponível: http://dx.doi.org/10.5935/1414-8145.20150038.

10. Freitas JS, Lima MVP, Santos JS, Lopes DR, Barbosa VM, Pachú CO. Consumo de álcool: influência familiar entre escolares. Biofarm. [Internet] 2014;10(3) [acesso em 23jan 2017]. Disponível: http://revista.uepb.edu.br/index.php/ biofarm/article/view/2605/1376.

11. Soccol KLS, Terra MG,Girardon-Perlini NMO, Ribeiro DB, de Silva CT, Camillo LA. O cuidado familiar ao indivíduo dependente de álcool e outras drogas. Rev. Rene. [Internet] 2013;14(3) [acesso em 26 set 2015]. Disponível: http:// www.revistarene.ufc.br/revista/index.php/revista/article/view/1104.

12. Machado ASM, Monteiro OS, Ribeiro LM, Guilhem D. Consumo de álcool por enfermeiros e implicações para o trabalho em saúde: revisão integrativa. Cogitareenferm. [Internet]2016;21(4) [acesso em 21 jun 2017]. Disponível: http://dx.doi.org/10.5380/ce.v21i4.45976. 
13. Kermode $\mathrm{M}$,Sono $\mathrm{CZ}$, Songput $\mathrm{CH}$, Devine A. Falling through the cracks: a qualitative study of HIV risks among women who use drugs and alcohol in Northeast India.BMC int. health hum. rights. [Internet]2013;13(9) [acesso em 23 Mar 2017]. Disponível: http://dx.doi.org/10.1186/1472-698X-13-9.

14.PiresFB, SchneiderDR. Projetosdevidaerecaídasempacientesalcoolistas. Arq. bras. psicol. [Internet]2013;65(1) [acesso em 09 out 2015]. Disponível: http://pepsic.bvsalud.org/scielo.php?script=sci_arttext\&pid=S1809-52672013000100003.

15. Barbosa KKS, Rocha WS, Vieira KFL, Alves ERP, Leite GO, Dias MD. Concepções de usuários de crack acerca da droga. Rev. enferm. UFSM. [Internet]2015;5(2) [acesso em 07jun 2015]. Disponível: http://dx.doi.org/10.5902/2179769213474.

16. Soccol KLS, Terra MG, Ribeiro DB, Mostardeiro SCTS, Teixeira JKS, Souto VT et al. Sobrecarga financeira vivenciada por familiares cuidadores de indivíduos dependentes químicos. Rev. enferm. UFSM. [Internet] 2014;4(3) [acesso em01Set 2015]. Disponível: http://dx.doi.org/10.5902/2179769211264.

17. Sousa PF,Ribeiro LCM, de Melo JRF, Maciel SC, Oliveira MX. Dependentes químicos em tratamento: um estudo sobre a motivação para mudança. Temas psicol. [Internet]2013;21(1)[acesso em 08 out 2015]. Disponível: http:// pepsic.bvsalud.org/scielo.php?script=sci_arttext\&pid=S1413-389X2013000100018.

18. doNascimento LTR, de Souza J, Gaino LV. Relacionamento entre familiar e usuário de álcool em tratamento em um centro de atenção psicossocial especializado. Texto contexto- enferm. [Internet]2015;24(3) [acesso em 24jan 2017]. Disponível: http://dx.doi.org/10.1590/0104-07072015003610013.

19. Sofin Y,Danker-Hopfe H, Gooren T, Neu P.Predicting Inpatient Detoxification Outcome of Alcohol and Drug Dependent Patients: The Influence of Sociodemographic Environment, Motivation, Impulsivity and Medical Comorbidities. J Addict. [Internet] 2017;2017[acesso em 23 mar 2017]. Disponível:http://dx.doi.org/10.1155/2017/6415831.

20. Ribeiro CT, Fernandes AH. Tratamentos para usuários de drogas: possibilidades, desafios e limites da articulação entre as propostas da redução de danos e da psicanálise. Analytica. [Internet]2013;1(2) [acesso em 09 set 2015]. Disponível: http://pepsic.bvsalud.org/scielo.php?script=sci_arttext\&pid=S2316-51972013000100003.

21. Croxford A,Notley CJ,Maskrey V,Holland R, Kouimtsidis C. An exploratory qualitative study seeking participant views evaluating group Cognitive Behavioral Therapy preparation for alcohol detoxification. Journal of Substance Use. [Internet]2015;20(1) [acessoem 23 mar 2017]. Disponível: http://dx.doi.org/10.3109/14659891.2014.894590.

22. Running BU, Beals J,Novins DK, Manson SM. Alcohol detoxification completion, acceptance of referral to substance abuse treatment, and entry into substance abuse treatment among Alaska Native people.Addict. behav. [Internet]2017;65[acesso em 23 Mar 2017].Disponível: https://doi.org/10.1016/j.addbeh.2016.09.009.

23. Reis GA, de Góis HR, Alves MS, Partata AK. Alcoolismo e seu tratamento. Revista Científica do ITPAC. [Internet] 2014;7(2) [acesso em 24 jan 2017 ]. Disponível: http://www.itpac.br/arquivos/Revista/72/4.pdf.

24. Jomar RT, Abreu AMM, Griep RH. Padrões de consumo de álcool e fatores associados entre adultos usuários de serviço de atenção básica do Rio de Janeiro, RJ, Brasil. Ciênc. saúde coletiva. [Internet] 2014;19(1) [acesso em 24jan 2017]. Disponível: http://dx.doi.org/10.1590/1413-81232014191.2009. 